\title{
Como obtener el máximo beneficio de los fondos de investigación científica: el ejemplo de Costa Rica (logros y retos FEES 2006-2016)
}

\author{
Lizette Brenes Bonilla' \& Julián Monge-Nájera² \\ 1. Universidad Estatal a Distancia, Vicerrectoría de Investigación, 2050 San José, Costa Rica; Ibrenesb@gmail.com \\ 2. Universidad Estatal a Distancia, Vicerrectoría de Investigación, Laboratorio de Ecología Urbana, 2050 San José, Costa Rica; \\ julianmonge@uned.ac.cr, julianmonge@gmail.com
}

Recibido 05-XI-2018 • Corregido 16-I-2019 • Aceptado 30-I-2019

\begin{abstract}
How to obtain the maximum benefit from scientific research funds: the example of Costa Rica (achievements and challenges, FEES 2006-2016)". Introduction: Costa Rica is the country with the most developed scientific community in Central America. In 2005, Costa Rican public universities, coordinated by a Council of Public Universities (CONARE), decided that, instead of working in isolation, they would pool resources. These financed joint research to solve environmental problems and support groups in disadvantage, like women and minorities. Objective: to examine the results after the first ten years of the program with emphasis on who received the funds and what products were obtained. Methods: we surveyed project coordinators and obtained replies from about half of them (i.e. the coordinators of 71 projects answered). Results: We found that $60 \%$ of research funds benefited disadvantaged populations (i.e. those in coastal and border areas) and that $54 \%$ of researchers who received funds were female. Survey projects produced 1388 scientific articles and 883 practical products, such as community workshops and commercial start-ups (estimate: over 2700 articles and 1700 practical products for the whole set of projects). Conclusion: These projects have been highly productive, have benefited women and underserved communities, led to lasting collaboration among researchers from different institutions, and are an example for other small countries with limited research funds.
\end{abstract}

Key words: fund distribution, research and development, women in science, science for underserved communities, academic cooperation in the tropics, impact of science, CONARE.
RESUMEN: Introducción: Costa Rica es el país con mayor desarrollo científico en América Central. En 2005, las universidades públicas costarricenses, coordinadas mediante un Consejo Nacional de Rectores decidieron que, en lugar de trabajar aisladamente, reunirían recursos para financiar investigaciones conjuntas para resolver problemas ambientales y apoyar a grupos en desventaja, como mujeres y minorías. Objetivo: examinar los resultados de la primera década del programa, con énfasis en quién recibió los fondos y cuáles productos se generaron. Métodos: encuestamos a los coordinadores de proyectos y obtuvimos respuestas de aproximadamente la mitad de ellos ( $N=71$ proyectos). Resultados: el $60 \%$ de los fondos de investigación beneficiaron a poblaciones desfavorecidas (es decir, las de las zonas costeras y fronterizas) y el $54 \%$ de los investigadores que recibieron fondos eran mujeres. Los 71 proyectos que completaron la encuesta produjeron 1388 artículos científicos y 883 productos prácticos como talleres comunitarios y nuevas empresas (estimación: más de 2700 artículos y 1700 productos prácticos en total). Conclusión: estos proyectos han sido altamente productivos: han beneficiado a mujeres y comunidades marginadas; han llevado a una colaboración duradera entre investigadores de diferentes instituciones, y son un ejemplo para otros países pequeños con fondos de investigación limitados.

Palabras clave: distribución de fondos, investigación y desarrollo, mujeres en la ciencia, ciencia para comunidades marginadas, cooperación académica en los trópicos, impacto de la investigación, CONARE.
La producción científica centroamericana se encuentra en crecimiento, aunque existen grandes diferencias entre países y un dominio visible de los hombres tanto por publicaciones como por nivel académico e impacto (Brenes-Bonilla, 2018). Por ejemplo, si solo se toman en cuenta revistas indexadas en los EEUU, la productividad per cápita de Costa Rica o Panamá es diez veces mayor que la de cualquiera de sus vecinos centroamericanos, aunque en el caso de Panamá hay un sesgo por la presencia del Smithsonian Tropical Research Institute, un poderoso centro extranjero de investigación (MongeNájera \& Ho, 2012, 2016).

Las diferencias también se dan en cuanto a temas investigados: Guatemala, Honduras y Nicaragua hacen el énfasis en salud y agricultura típico de los países poco desarrollados; sin embargo, es posible que este patrón sea diferente en la literatura excluida del Science Citation Index (Monge-Nájera \& Ho, 2017a, b; Monge-Nájera \& Ho, 2018). 
En los países centroamericanos hay una dependencia científica de los Estados Unidos y Europa, cuyos cientíicos dirigen la mayoría de los equipos de investigación de alta visibilidad, aunque el efecto es menos marcado en Costa Rica (Monge-Nájera \& Ho, 2012). También presentan condiciones desventajosas Guatemala, por las pocas revistas científicas propias (datos Latindex analizados en Monge-Nájera \& Ho, 2018), y Nicaragua, por la baja participación femenina en la ciencia y en la tecnología (Monge-Nájera \& Ho, 2017a). El Salvador es un caso atípico porque su producción principal se concentra en las ciencias sociales (Monge-Nájera \& Ho, 2017c), por lo que posiblemente allí la presencia femenina sea mayor, ya que las mujeres suelen dominar numéricamente las ciencias sociales (Cislak, Formanowicz, \& Saguy, 2018)

Las universidades públicas costarricenses se encuentran agrupadas en el Consejo Nacional de Rectores (CONARE). Los investigadores de estas universidades pueden acceder a un fondo especial concursable, conocido como Fondo del Sistema, el cual forma parte del Fondo Especial para la Educación Superior (FEES). Además, existen otros fondos para la investigación dados por otros entes gubernamentales, por ejemplo, el Consejo Nacional para Investigaciones Científicas y Tecnológicas (CONICIT) o por entes privados, por ejemplo, Asociación Costa Rica por Siempre (detalles en conicit.go.cr; micit.go.cr y ricyt.org).

Oficialmente, desde el 2005, el Fondo del Sistema pretende aumentar la cooperación, la productividad y la integración de los talentos y recursos de las universidades públicas para enfrentar los retos de Costa Rica (conare. ac.cr). Para saber si el experimento ha tenido éxito, en este artículo analizamos quienes recibieron los recursos y cuáles beneficios produjeron estos proyectos en sus primeros diez años de funcionamiento.

\section{MÉTODOS}

Las vicerrectorías de investigación de las cuatro universidades públicas participantes enviaron una encuesta digital a todos los coordinadores de proyecto $(n=157)$ beneficiados con fondos en esos diez años, indicando que su cooperación sería tomada en cuenta en futuras solicitudes. Obtuvimos respuesta de los coordinadores de 71 proyectos. Un obstáculo para obtener respuestas fue que, tras diez años, algunos coordinadores ya no estaban disponibles por cambio de empleo, retiro o fallecimiento. Analizamos los datos con Excel para generar gráficos y buscar visualmente las tendencias a lo largo del tiempo. En el Apéndice Digital 1 se presentan en detalle los gráficos por total y los cocientes por proyectos.
Ética, conflicto de intereses y declaración de financiamiento: los autores declaran haber cumplido con todos los requisitos éticos y legales pertinentes, tanto durante el estudio como en el manuscrito; que fueron temporalmente parte de las comisiones que evaluaban los proyectos a financiar, y que todas las fuentes financieras se detallan plena y claramente en la sección de agradecimientos. Asimismo, están de acuerdo con la versión editada final del documento. El respectivo documento legal firmado se encuentra en los archivos de la revista.

\section{RESULTADOS}

El presupuesto invertido en el Fondo del Sistema en el periodo 2006-2016, ha variado entre 200 millones y 1 800 millones de colones, con tendencia histórica hacia una disminución (Apéndice 1).

En este apartado presentamos el detalle de quienes fueron beneficiados con los fondos (a nivel de investigadores y comunidades) y cuáles fueron los productos de ésta, la mayor inversión en investigación científica de la historia de Costa Rica.

Personal beneficiado: A nivel de investigadores participantes, la institución más beneficiada fue la Universidad de Costa Rica con 215 personas, seguida de la Universidad Nacional con 191, el Instituto Tecnológico de Costa Rica con 84 y finalmente la Universidad Estatal a Distancia con 57. La Universidad Estatal a Distancia no contaba con sistema de investigación durante los primeros cinco años de existencia de este fondo, y por lo tanto solo participaba marginalmente, a menudo como encargada de imprimir folletos o libros, si el proyecto llegaba a producirlos, lo cual no siempre ocurría.

En cuanto al género, predominaron las mujeres (572 mujeres beneficiadas, así: 285 Investigadoras y 287 estudiantes); los hombres estuvieron ligeramente por debajo de la paridad (485 hombres: 276 investigadores, 209 estudiantes). El 39\% de las coordinaciones estuvieron a cargo de mujeres, aumentando en años recientes (Apéndice 1).

Comunidades beneficiadas: La mayoría de las comunidades beneficiadas están en las zonas con mayor necesidad de desarrollo, con un $60 \%$ de los proyectos beneficiándolas (zonas costeras 30\% y zonas fronterizas $30 \%)$. La Gran Área Metropolitana recibió beneficios del $40 \%$ de los proyectos. 
Productos: Se produjeron 1388 artículos científicos y 883 productos prácticos como talleres comunitarios y nuevas empresas (estimación: más de 2700 artículos y 1700 productos prácticos en total al incluir en el cálculo los proyectos que no respondieron la encuesta).

Los resultados transferidos a los grupos meta fueron principalmente reuniones con sectores y comunidades $(\mathrm{N}=337)$, cercanamente seguidos de participación en eventos científicos $(\mathrm{N}=322)$ y otros productos varios ( $N=132)$. La presencia en los medios de comunicación también fue importante, con 137 reportajes y entrevistas (Apéndice 1).

Los artículos en revistas indexadas (así calificadas por quienes llenaron la encuesta) fueron principalmente en revistas de América Latina ( $\mathrm{N}=147)$ seguido de publicaciones en revistas indexadas de los EEUU ( $N=90)$, Europa $(\mathrm{N}=79)$ y otras regiones $(\mathrm{N}=46)$. Los encargados de los proyectos también informaron haber publicado 45 artículos en "revistas no indexadas".

Las otras publicaciones también fueron numerosas: 89 folletos, 74 capítulos de libro, 60 mini folletos y volantes, 23 libros y 23 manuales (Apéndice 1).

En el área docente, hubo 213 cursos beneficiados con el proyecto, produciéndose 242 trabajos finales de graduación, 201 tesis de grado y 145 tesis de postgrado (Apéndice 1).

A nivel comercial, se lograron 52 productos (principalmente productos agrícolas, como mermeladas y otros alimentos procesados), 30 procesos (principalmente procesos agro-industriales mejorados) y 5 patentes (Apéndice 1).

Además, 67 investigadores/as continuaron colaborando entre sí tras acabar el proyecto, lo que implica un alto logro en el objetivo de lograr colaboración científica interinstitucional.

Dificultades: El programa también tuvo aspectos débiles. Después del 2010 los fondos asignados por las universidades comenzaron a decrecer, lo que obligó a dar cada vez menos fondos por proyecto, reduciéndose al final a unos $\$ 70000$ por proyecto (Apéndice 1). En paralelo con esto, el interés por participar en estos proyectos disminuyó y desde ese año se estancó el número de proyectos recibidos en concurso.

Además, cerca de diez proyectos por año no lograron concluir dentro del calendario, obligando a conceder prórrogas, siendo 2015 el peor año en ese sentido (Apéndice 1). Temáticamente, las humanidades casi no han logrado presentar proyectos con la calidad necesaria, por lo que la distribución temática ha sido dominada por las llamadas ciencias duras y la tecnología: 43 proyectos en salud, 37 en ciencias naturales, 22 en agricultura, 21 en tecnología electrónica e industrial, 12 en ciencias sociales y únicamente tres en humanidades (Apéndice 1). La colaboración internacional es mínima y se da fundamentalmente con otros países del continente (siendo los EEUU particularmente importantes) y con Europa Occidental.

\section{DISCUSIÓN}

Dos requisitos éticos básicos en la distribución de fondos de investigación son que se realice equitativamente y que produzca el mayor beneficio posible para la sociedad. Sin embargo, ambos requisitos pueden llegar a ser incompatibles, pues en algunos casos distribuir fondos por igual a todas las instituciones podría resultar en menos fondos para los equipos que producirían el mayor beneficio real para la comunidad (Emanuel et al., 2004; Ballantyne, 2010). Por lo tanto, la distribución debe buscar un equilibrio entre equidad, calidad y beneficio real.

En el caso del FEES las universidades pequeñas han recibido fondos proporcionalmente mayores que los que corresponderían a su tamaño (CONARE, 2018).

En muchos países se busca incrementar la presencia femenina en los equipos de investigación científica, con proyectos como STEM4girls (http://www.stem4girls.org/); y Monge-Nájera \& Ho (2017a) han advertido sobre la escasa participación de mujeres en países como Nicaragua (de nuevo, con datos limitados a las publicaciones del Science Citation Index, que para nada no es representativo de la producción centroamericana). Sin embargo, la situación en Costa Rica es más cercana a la paridad, con $43 \%$ de investigadoras y un crecimiento mayor de mujeres (12\%) que de hombres (8\%) en las carreras científicas (Umaña \& Vargas, 2018). Nuestro análisis calza con estos datos y demuestra que, en el FEES, la participación de las mujeres (54\%) supera a la de los hombres, lo que indica un resultado equitativo en este campo. Esta tendencia la encontramos confirmada también en los datos publicados por CONARE (2018: https://bit.ly/2G9jUGv). Pareciera que una vez más se está invirtiendo el patrón hacia la tendencia original descrita por Jason Farago, y hoy fundamentalmente desconocida:

"Hasta mediados del siglo XIX -cuando las ciencias se profesionalizaron- eran las mujeres quienes debían estar en el laboratorio, o entre los especímenes geológicos y zoológicos. En aquella época, eran las humanidades las que eran vistas como campos puramente masculinos" (Farago, 2018). 
Aunque no suele mencionarse, los hombres están en marcada desventaja como estudiantes universitarios en ciencias de la salud, ciencias económicas, artes y letras, ciencias sociales y educación (Apéndice 2, elaborado por Ligia Bermúdez a partir de datos oficiales del CONARE). Es en ciencias básicas e ingeniería, donde las mujeres deben aumentar, y existe casi una paridad en recursos naturales (Apéndice 2).

Las preocupaciones válidas en investigación incluyen el que no atiendan los problemas más acuciantes de una sociedad (Ellis \& Levy, 2008) y el de que se archiven en forma de literatura gris que nunca produzca un beneficio real (Jiménez-Medina, 2014). Nuestros hallazgos indican que ese temor no calza con este experimento costarricense, dados la predominancia de proyectos FEES en comunidades poco desarrolladas, y la alta productividad de los proyectos, medida en productos comerciales, actividades comunitarias, artículos científicos, libros, tesis y cursos. Además, $70 \%$ de los cantones con bajo desarrollo humano están en zonas costeras y fronterizas, y forman los conglomerados de mayor desigualdad (Brenes-Bonilla et al., 2015), por lo que resulta socialmente significativo que los proyectos FEES beneficiaran prioritariamente a esos cantones

La colaboración científica es valiosa para aumentar la calidad e impacto de la investigación científica, y en América Latina tiende a ser insuficiente a nivel interno de cada país (Russell et al., 2007). Aunque en el caso de Costa Rica no se ha documentado, la experiencia personal de los autores de este informe es que, a fines del siglo 20, había poco ambiente para colaboración entre universidades públicas. En nuestra encuesta, 67 de los 71 coordinadores informaron que siguieron colaborando interinstitucionalmente tras acabar el proyecto, cumpliendo uno de los objetivos del FEES.

Dos logros que quedan pendientes en esta primera década. Primero, aumentar el financiamiento a los proyectos. Segundo, elevar el nivel de calidad de las propuestas en humanidades y ciencias sociales, para que sean más competitivos y reciban mayor financiamiento, aunque eso requerirá cambios grandes en ese campo tradicionalmente conservador en cuanto a enfoques y métodos (Monge-Nájera, 2011).

En conclusión, los proyectos FEES han sido en general exitosos y muy productivos, han beneficiado de manera justa a mujeres y comunidades con mayor necesidad, y representan un ejemplo para otros países pequeños que quieran obtener el máximo provecho de sus limitados fondos de investigación.

\section{AGRADECIMIENTOS}

Agradecemos la colaboración de Cristina Alvarado, Ligia Bermúdez, Maribel Jiménez, Silvia Sáenz, Sharlín Sánchez, Viviana Salgado, y Maynor Vargas en la recopilación y análisis de los datos; de Elena Vargas en la preparación del manuscrito, y de un(a) revisor(a) anónimo(a) que hizo aportes particularmente valiosos para mejorar el manuscrito.

\section{REFERENCIAS}

Ballantyne, A. J. (2010). How to do research fairly in an unjust world. The American Journal of Bioethics, 10(6), 26-35. DOI: $10.1080 / 15265161.2010 .482629$

Brenes-Bonilla, L. (2018). Investigación y Posgrado en América Central y República Dominicana. (Informe técnico). San José, Costa Rica: Consejo de Investigación del Sistema Centroamericano de Investigación y Posgrado.

Brenes-Bonilla, L., Bermúdez-Mesén, L., \& Bermúdez-Vargas, K. (2015). SME's semi-formality rate in Costa Rica: a clusters approach. Faedpyme International Review, 4(7), 4752. DOI:10.15558/fir.v4i7.98

Cislak, A., Formanowicz, M., \& Saguy, T. (2018). Bias against research on gender bias. Scientometrics, 115(1), 189-200. DOI:10.1007/s11192-018-2667-0

CONARE (Consejo Nacional de Rectores). (2018). Cuarto Informe de Ejecución Presupuestaria. San José, Costa Rica: Consejo Nacional de Rectores.

Ellis, T. J., \& Levy, Y. (2008). Framework of problem-based research: A guide for novice researchers on the development of a research-worthy problem. Informing Science: the International Journal of an Emerging Transdiscipline, 11, 17-34.

Emanuel, E. J., Wendler, D., Killen, J., \& Grady, C. (2004). What makes clinical research in developing countries ethical? The benchmarks of ethical research. The Journal of infectious diseases, 189(5), 930-937. DOI: 10.1086/381709

Farago, J. (2018, 26 de julio). Mushrooms, Magma and Love in a Time of Science. The New York Times, pp. C11.

Jiménez-Medina, J. A. (2014). Publicar o morir: divulgación de conocimiento en el campo editorial universitario, el caso colombiano. Dialéctica Libertadora, 6, 122-136.

Monge-Nájera, J. (2011). Darwin y su poco impacto en las ciencias sociales costarricenses. Cuadernos de Antropología, $21,1-6$.

Monge-Nájera, J., \& Ho, Y. S. (2012). Costa Rica Publications in the Science Citation Index Expanded: A bibliometric analysis for 1981-2010. Revista de Biología Tropical, 60(4), 1649-1661. DOI: 10.15517/rbt.v60i4.2158

Monge-Nájera, J., \& Ho, Y. S. (2016). Bibliometry of Panama publications in the Science Citation Index Expanded: 
publication type, language, fields, authors and institutions. Revista de Biología Tropical, 63(4), 1255-1266. DOI: 10.15517/rbt.v63i4.21112

Monge-Nájera, J., \& Ho, Y. S. (2017a). Bibliometrics of Nicaraguan publications in the Science Citation Index Expanded. Revista de Biología Tropical, 65(2), 643-655. DOI: $10.15517 /$ rbt.v65i2.23985.

Monge-Nájera, J., \& Ho, Y. S. (2017b). Honduras publications in the Science Citation Index Expanded: institutions, fields and authors. Revista de Biología Tropical, 65(2), 657-668. DOI: $10.15517 /$ rbt.v65i2.25893

Monge-Nájera, J., \& Ho, Y. S. (2017c). El Salvador publications in the Science Citation Index Expanded: subjects, authorship, collaboration and citation patterns. Revista de Biología Tropical, 65(4), 1428-1436. DOI: 10.15517/rbt. v65i4.28397
Monge-Nájera, J., \& Ho, Y. S. (2018). Guatemala articles in the Science Citation Index Expanded: bibliometry of subjects, collaboration, institutions and authors. Revista de Biología Tropical, 66(1), 312-320. DOI: 10.15517/rbt. v66i1.29875.

Russell, J. M., Ainsworth, S., del Río, J. A., Narváez-Berthelemot, N., \& Cortés González, H. D. (2007). Colaboración científica entre países de la región latinoamericana. Revista Española de Documentación Científica, 30(2), 180-198.

Umaña, P., \& Vargas, L. (2018, 10 de agosto). La participación de las mujeres continúa relegada en las ciencias. Noticias UCR. Recuperado de www.ucr.ac.cr/ noticias/2018/08/10/la-participacion-de-las-mujerescontinuarelegadaen-las-ciencias.html. 